\title{
Redo Surgery After Multiple Coronary Endarterectomy
}

\author{
ABSTRACT \\ Coronary endarterectomy was recognized as the most controversial method \\ in cardiac surgery due to high perioperative morbidity and mortality. These \\ arguments significantly decreased utilization of coronary endarterectomy in the \\ last two decades. We present a 65 y/o male with unstable angina and low ejection \\ fraction $(E F=28 \%) 15$ years after multiple coronary endarterectomy. The patient \\ was successfully treated with redo coronary bypass surgery and reverse heart \\ remodeling, and has remained stabile one year after surgery.
}

\section{Živojin S. Jonjev, Zoran Konstantinović, Vladimir Torbica, Milenko I. Rosić}

\author{
University of Novi Sad, Institute for \\ Cardiovascular Diseases of Vojvodi- \\ na, Clinic of Cardiovascular Surgery, \\ Sremska Kamenica, Serbia
}

\section{Corresponding Author:}

Živojin S. Jonjev, MD, PhD

Institute for Cardiovascular Diseases Institutski put 4

21204 Sremska Kamenica, Serbia

Phone: + 381642182367

Fax: +381216622059

E-mail: jonjevz@nscable.net
Coronary artery bypass grafting (CABG) has long been a standard procedure in patients with coronary artery disease. However, CABG with endarterectomy is one of the most controversial methods in cardiac surgery. ${ }^{1}$ High technical demands, long duration of the procedure, and consequent increased perioperative morbidity and mortality are arguments that discourage the use of coronary endarterectomy. ${ }^{2}$ End-stage coronary artery disease is usually complicated with heart remodeling and functional mitral and tricuspid regurgitation. ${ }^{3}$ In this report we present clinical findings and outcome in redo surgery 15 years after multiple coronary endarterectomies, complicated overtime with low ejection fraction (EF) and mitral and tricuspid insufficiency.

\section{Case Presentation}

The patient was a 64-year-old Caucasian male with chest pain and easy fatigue that progressively worsened eight month prior to admission. Patient's past medical history was positive for $17 \mathrm{y} / \mathrm{o}$ posteroinferior myocardial infarction, arterial hypertension, insulin independent diabetes mellitus, and family history of ischemic heart disease. In addition, 15 years ago patient underwent CABG surgery for unstable angina. Operative finding confirmed lower ejection fraction $(\mathrm{EF}=45 \%)$ and diffuse coronary artery disease. Extensive double endarterectomy of the right coronary artery (RCA) and left anterior descending artery (LAD) was performed, followed with double venous CABG. The endarterectomy was performed by closed and manual operative technique. The endarterectomized sequesters were 8.5 and $11 \mathrm{~cm}$ in length, respectively and were evacuated from the vessels by blunt dissection. The anastomosis were closed without patching and grafts were routinely flushed with cold crystalloid cardioplegic solution to evaluate distal runoff. ${ }^{2}$ Clinical outcome after the first surgery was good, and the patient was discharged from the hospital on the postoperative day \#10, without complications.

This time, on admission, physical exam and chest roentgenogram demonstrated signs of end-stage ischemic dilative cardiomyopathy (NYHA III/IV). Electrocardiogram confirmed sinus rhythm $(\mathrm{F}=76 / \mathrm{min})$, myocardial infarction of the posterior wall of the left ventricle, without signs of ischemia at rest. Cardiac catheterization studies showed bilateral graft stenosis (>90\%), good visualization of endarterectomized RCA and LAD territory (Figure 1a and 1b), and multiple coronary stenosis (total occlusion of the first diagonal artery $\left(\mathrm{D}_{1}\right),>90 \%$ stenosis of the ramus intermedius (RIM), and first obtuse marginal artery $\left.\left(\mathrm{CxOM}_{1}\right)\right)$. Transthoracic echocardiography showed significant mitral and tricuspid regurgitation (III/IV degree) due to extensive heart remodeling, and $\mathrm{EF}=\mathbf{2 8} \%$.

The patient underwent elective redo heart surgery with significantly increased perioperative risk (EuroSCORE [E$\log ]=8.12$; STS score $[\mathrm{E}-\log ]=12.7$ ). Intraoperative transesophageal echocardiography was performed using the 
Toshiba SSH-140-A and Omniplane 5.0 MHz probe, before and after surgery.

After heart exposure, using cardiopulmonary bypass, we induced cardiac arrest and standard myocardial protection. Myocardial revascularization was successfully achieved with triple bypass using left internal mammary artery to $\mathrm{D}_{1}$, and double venous CABG to RIM and CxOM territory. By mini transseptal approach, modified stitch annuloplasty of mitral and tricuspid valves was performed in addition to myocardial revascularization ${ }^{3}$. Postoperative course of the patient was uneventful. He was discharged from the hospital on postoperative day $\# 9$, and has remained stabile one year after surgery.

\section{Discussion}

In the past, the worldwide incidence of coronary endarterectomy ranged from $1.9 \%$ to $17.9 \% .^{1,2}$ However, controversy in perioperative morbidity and mortality significantly decreased utilization of this method in many respectable cardiac centers. The same situation happened in our institution regardless of our experience and reputation in this field. Questionable indications, unstandardized operative technique, higher incidence of early thrombosis and unreported long-term results due to the lack of late angiography raised many intriguing questions $\mathbf{s}^{1,2}$.

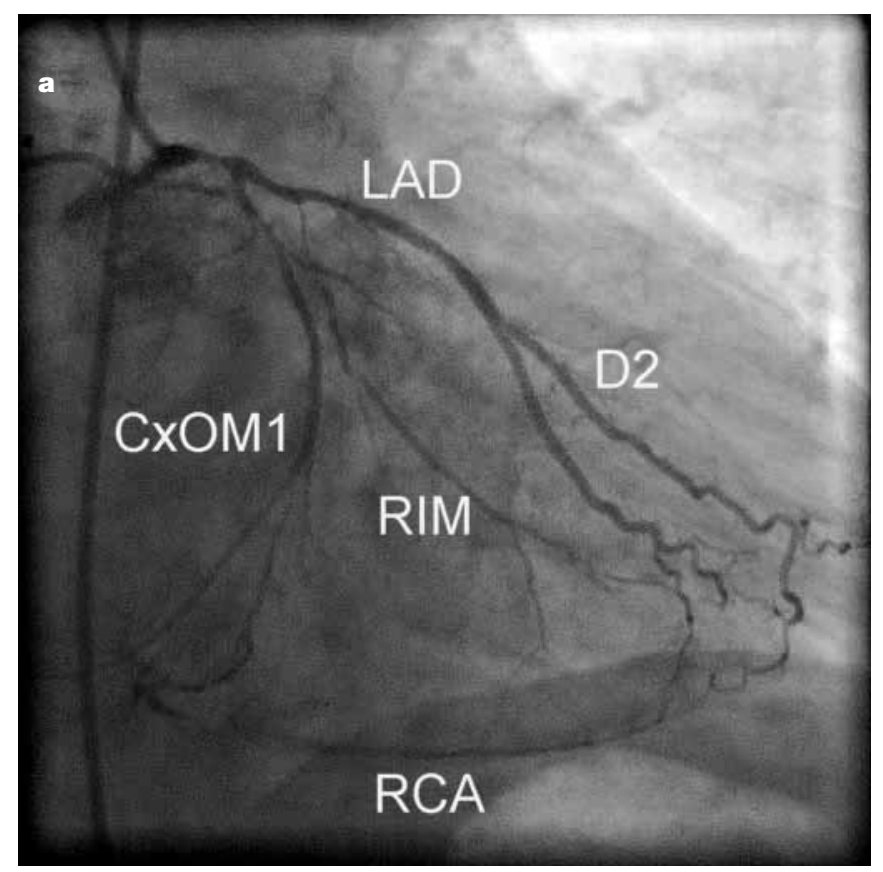

At present, the number of patients with diffuse and distal coronary disease is high, and is still increasing. ${ }^{2}$ Recently published literature emphasized that high incidence of "non-graftable arteries" is not only related to the natural progression of the disease, but to the advances in coronary angioplasty, as well. For that reason, preoperative decision-making process and standardization in CABG surgery is even more difficult.

When a patient presents himselfe/herselfe in the advanced stages of coronary atherosclerosis, or has unsatisfactory results after multiple stenting, three ways of treatment have been proposed: incomplete bypass surgery, transmyocardial laser revascularization or angiogenic growth factor therapy. ${ }^{3}$ Nevertheless, all these options are wrought with problems such as low freedom from cardiac events requiring hospital admission, and associated high costs of treatment. The second option is to perform a complete myocardial revascularization with one of the most controversial and almost forgotten method: coronary endarterectomy. Long-term results after extensive coronary endarterectomy are usually not very well presented in the literature. However, good angiographic visualization of endarterectomized coronary arteries, and possibility for redo surgery 15 years later suggests to us to keep this method in the armamentarium of surgical treatment and research.

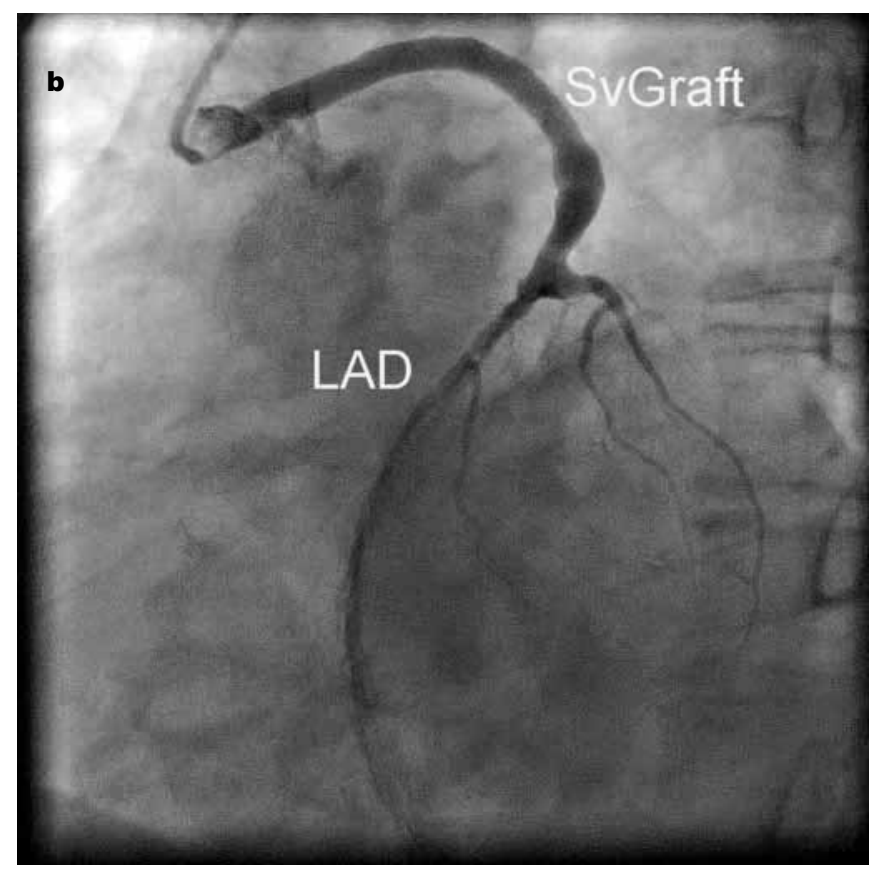

Figure 1. Preoperative and Postoperative Coronarography.

a. Coronarography of the left coronary artery 15 years after extensive double endarterectomy. Findings show good visualization of endarterectomized LAD territory, retrograde filling of the RCA, and significant coronary stenosis of the ramus intermedius (RIM), and first obtuse marginal artery ( $\left.\mathrm{CxOM}_{1}\right)$. The first diagonal artery was proximally occluded and identified intraoperatively. RCA-right coronary artery; LAD-left anterior descending artery; D1-first diagonal artery; RIM-ramus intermedius artery; CxOM1-first obtuse marginal artery.

b. Coronarography of the saphenous venous LAD graft is showing good visualization of endarterectomized LAD territory 15 years after the first surgery. LAD-left anterior descending artery, SvGraft - saphenous venous graft. 
The second issue in end-stage coronary disease is heart remodeling and consequent functional mitral and tricuspid insufficiency, such as that seen in our patient. Recent reports indicated that a reduction in mitral annulus area (MAA) significantly changes the sphericity of the left ventricle 24 months after surgery, and could have an effect of "slow auto-remodeling" of the left ventricle ${ }^{3,4}$. Because of the considerable interdependence of both ventricles, mitral and tricuspid insufficiency should be repaired concomitantly with the same surgery. In the presented case our method of choice was modified stitch annuloplasty of mitral and tricuspid valves (RADO procedure) ${ }^{3}$. Our personal experience and recently published literature suggest us that stich anuloplasty in cases with low mitral valve tenting area (coaptation depth $<1 \mathrm{~cm}$ ) is quite acceptable ${ }^{3,5}$. This simple maneuver performs reduction and restores normal shape of mitral valve, avoids annular rigidity commonly described after rigid ring annuloplasty and permits three-dimensional movement of the valve. Using this approach we demonstrated that reconstruction of valvular abnormality in addition to CABG improved left ventricular function, and could be used for "reverse" heart remodeling. Thus, it should not be recognized as a simple valve repair but ventricular repair as well.

We may conclude that redo surgery after multiple coronary endarterectomy is still a challenge. However, it could be successfully performed even in patients with end-stage ischemic dilated cardiomyopathy. We report a case of a successful redo CABG surgery combined with redo proce- dure 15 years after multiple coronary endarterectomy. The patient survived operation without postoperative complications and stayed well and without cardiac events for a year after surgery. Therefore, we propose redo procedure combined with CABG as a bridge to recovery or even destination therapy in selective patients with ischemic dilative cardiomyopathy.

\section{Acknowledgements}

The authors wish to thank Ninoslav D. Radovanović, MD, for his contribution in preparing this manuscript.

\section{References}

1. Sundt TM, III, Camillo CJ, Mendeloff EN et al. Reappraisal of coronary endarterectomy for the treatment of diffuse coronary artery disease. Ann Thorac Surg 1999; 68:1272-7.

2. Jonjev ZS, Nicin S, Mujovic V et al. Prostacyclin Reduces Incidence of Myocardial Damage After Coronary Endarterectomy. Ann Thorac Surg 2004; 78:1299-303.

3. Jonjev ZS, Mijatov M, Fabri M et al. Systematic reductive annuloplasty of the mitral and tricuspid valves in patients with end-stage ischemic dilated cardiomyopathy. J Card Surg 2007; 22:111-6.

4. Cohn LH, Rizzo RJ, Adams DH et al. The effect of pathophysiology on the surgical treatment of ischemic mitral regurgitation: operative and late risks of repair versus replacement. Eur J Cardiothorac Surg 1995; 9:568-74.

5. Fundaro P, Tartara PM, Villa E et al. Mitral Valve Repair: Is There Still a Place for Suture Annuloplasty? Asian Cardiovasc Thorac Ann 2007; 15:351-8. 